\section{Non-smoking woman with adenocarcinoma of the lung, IV stage with ROS1 mutation and acquired thrombophilia}

\author{
Immanuels Taivans ${ }^{1,2 *}$, Natalja Senterjakova ${ }^{1,2}$, Viktors \\ Kozirovskis ${ }^{1,2}$, Gunta Strazda ${ }^{1,2}$, Jurijs Nazarovs ${ }^{2}$ and Valentina \\ Gordjusina ${ }^{1}$
}

${ }^{1}$ Faculty of Medicine, University of Latvia, Riga, Latvia

${ }^{2}$ Pauls Stradins Clinical University Hospital, Riga, Latvia

\section{Abstract}

Despite the fact, that lung cancer is more common among older smoking men, however it may also develop among young women without a smoking anamnesis. We report here a history of a non-smoking woman, 40 years old, with a diagnosis of lung adenocarcinoma at IV stage. Despite the fact, the woman received three lines of palliative chemotherapy, the disease progressed. After the sample of the tumor was tested by genetic approach, ROS1 mutation was detected, and the patient was treated with a ROS1 inhibitor, Crizotinib. Sharp improvement was observed already after the first week of treatment. After one-month adenocarcinoma shrink, and specific supraclavicular lymph nodes disappeared. Unfortunately, due to problems with financing the treatment was stopped, after what the disease began to progress rapidly, and the patient died after a month due to brain metastasis. This case is noteworthy also because the patient was first diagnosed a thrombophilia with thrombi present in deep calf veins, left heart ventricle and lungs Adenocarcinoma was discovered occasionally when during video-assisted thoracoscopic surgery biopsy specimen was taken from suspicious mass in the lower lobe of the right lung. This story reminds us that lung carcinoma may start with a paraneoplastic syndrome, like thrombophilia as in this case and finding of adenocarcinoma of the lung in young, nonsmoking persons is indicative for possible ROS1 gene mutation. In such cases early treatment with ROS1 protein-tyrosine kinase inhibitors should be started as soon as possible.

\section{More Information}

*Address for Correspondence: Immanuels Taivanss, Faculty of Medicine, University of Latvia, Riga, Latvia, Tel: +371 29428 136; Email: taivans@latnet.Iv

Submitted: July 13, 2021

Approved: August 03, 2021

Published: August 04, 2021

How to cite this article: Taivans I,

Senterjakova N, Kozirovskis V, Strazda G, Nazarovs J, et al. Non-smoking woman with adenocarcinoma of the lung, IV stage with ROS1 mutation and acquired thrombophilia. J Pulmonol Respir Res. 2021; 5: 064-072. DOI: 10.29328/journal.jprr.1001027

Copyright: @ 2021 Taivans I, et al. This is an open access article distributed under the Creative Commons Attribution License, which permits unrestricted use, distribution, and reproduction in any medium, provided the original work is properly cited.

Keywords: Adenocarcinoma of the lung; ROS1 mutation; Crizotinib; Acquired thrombophylia

Check for updates

OPEN ACCESS

\section{Introduction}

Lung cancer is one of the most common malignant tumors. It was primarily diagnosed in 2.2 million patients and 1.8 cases of death were estimated in 2020 [1]. It is mostly detected in 50-60 years old smoking men [2,3]. However, morbidity rate among women is growing $[1,4]$.

Development of lung cancer is associated with different gene mutations. Among them ROS1 rearrangements are found in $1 \%-2 \%$ cases of lung adenocarcinoma [6]. In the study of 727 lung adenocarcinomas from patients with stage IV disease, who were positive for ROS1 fusions was found that this mutation was more common in non-smoking females with advanced disease compared to patients with operable disease. The mean age at diagnosis was significantly lower in patients with advanced disease [7,8]. The finding of ROS1 mutation in advanced disease means that chromosomal mutations yielding fusion genes with ROS1 gene occur in late stages of tumor development.

ROS1 gene is located on chromosome 6 (6q22) and encodes the tyrosine kinase transmembrane receptor which consists of an intracellular C-terminal domain and an extracellular $\mathrm{N}$-terminal domain. Because a ligand for ROS1 receptor has not been found, the physiological function of this receptor is not known [9]. However, the extracellular domain of the receptor was studied and it is thought that the N-terminal domain is responsible for cellular attachment. Normal ROS1 protein is expressed in the kidney, cerebellum, peripheral neural tissue, stomach, small intestine, and colon, however it is not found in lung tissue [6].

ROS1 gene is vulnerable to different intrachromosomal or 
interchromosomal rearrangements that causes gene fusion, for examples, CD74-ROS1, SDC4-R0S1, EZR-ROS1, SLC34A2ROS1, TPM3-ROS, LRIG3-ROS, KDELR2-ROS1, CCDC6-ROS1. Up to date more than 20 fusion partners are found 10]. These fusion genes encode for oncoproteins which activate signaling pathways like MAPK and PI3K/AKT that are critical for carcinogenesis and progression, [11,12]. ROS1 gene mutation can be identified by such methods as fluorescence in situ hybridization (FISH), immunohistochemistry, real-time polymerase chain reaction (RT-PCR) and next generation sequencing (NGS) [13]. In recent years several new ROS1 fusion genes with oncogenic properties are discovered.

Treatment with a tyrosine kinase inhibitor, for example Crizotinib, gives good results practically in all ROS1 fusion gene cases. Crizotinib inhibits the ATP-binding to ALK and ROS1 receptors, suppressing the autophosphorylation of the receptors and arresting the signal transfer from receptor to cytoplasm $[14,15]$.

Microscopic studies have revealed that many adenocarcinomas are heterogeneous and consist of two or more subtypes. $80 \%$ of all lung adenocarcinomas are classified as a mixed ones. Adenocarcinomas histologically range from well-differentiated tumors with clear glandular elements to papillary formations resembling other papillary carcinomas and to solid masses with small amounts of mucusproducing glands and cells. Tumor cells have a large volume of cytoplasm, round or oval nuclei and well-visible nucleoli. Fibrosis is often observed. Even though adenocarcinoma can develop in connective tissue, often connective tissue or scars result from a desmoplastic reaction caused by the tumor itself. Macroscopically, adenocarcinoma often looks like a peripheral, subpleural node or mass, usually caused by the pleural retraction $[16,17]$.

Lung adenocarcinomas with ROS-1 mutations frequently are accompanied by thromboembolic paraneoplastic syndrome. In wide meta-analysis of 21,156 patients' cases with non-small cell lung carcinoma the group with ROS1 rearrangements had the highest incidence of VTE - 37\%. ALK rearrangements were associated with second highest VTE risk-27\%, while there was no significant relation observed between VTE risks and EGFR or KRAS mutations [18]. Analysis From a Phase II, Prospective, Multicenter, Two-arms Trial (METROS) study has shown even higher VTE incidence in ROS1 mutation positive patients reaching 41\%. Pulmonary embolism was observed in $46.4 \%$, deep vein thrombosis in $39.2 \%$, renal vein thrombosis in $7.1 \%$, internal jugular thrombosis in 3.5\%, and peripheral inserted central catheterrelated thrombosis-in 3.5\% among studied VTE patients. It was concluded that the incidence of VTE is 3- to 5-fold higher in patients harboring ROS1-rearrangment compared to the general population with NSCLC [19].

Chinese study of 5,767 NSCLC cases affirmed 41\% VTE rate in ROS1 mutation and 30\% rate in ALC mutations [20]. Taken together these data show that thrombophilia as manifestation of paraneoplastic syndrome depends on particular oncogenic alteration in the tumor's genome.

Tumor cells produce and secrete tissue factor, procoagulant/fibrinolytic substances, inflammatory cytokines that activate the blood clotting system. Together with factor VIIa, tissue factor catalyzes the conversion of inactive factor X into active protease Xa. Procoagulants-calciumdependent cysteine proteases, independent of VIIa and tissue factor, directly activate factor X. Tumor cells enhance the development of thrombosis by releasing plasminogen activator inhibitors and inflammatory mediators that affect the production of tissue factor $[18,19]$. It is known that oncogene and tumor suppressor gene-mediated transformation, as MET and K-RAS over-activation, loss of PTEN, EGFR mutation and p53 deficiency, can affect one of the intracellular signal pathways leading to activation of the blood coagulation system, platelet function or fibrinolysis suppression [23]. It has been found that the mutations of the Ras, p53, PTEN genes induce overexpression of the tissue factor, which can cause not only hypercoagulation, but also significantly affect tumor angiogenesis [24].

The purpose of this publication is to reveal the circumstances that may contribute to rapid spread of tumor and fatal outcome, as well as to justify optimal treatment strategies in similar cases.

\section{Clinical case report}

A 39-year-old patient was transferred from regional hospital to Pauls Stradins Clinical University Hospital, Riga, Latvia (P. Stradins CUH) on $15^{\text {th }}$ of January 2016 due to suspicions of acute myocarditis, acute pericarditis, and heart failure. Since the beginning of December 2015, the patient complained on non-productive cough, after the Christmas she also felt the sense of pressure in the chest and dyspnea while climbing the stairs. The woman had normal body temperature at home. Antibiotics were prescribed during an ambulatory care visit. Dyspnea progressed and became more severe in supine position and patient was hospitalized to regional hospital on $4^{\text {th }}$ January 2016. Chest radiography was performed which revealed the hydrothorax and lung congestion. Pleural effusion was revealed also during ultrasonographic examination. The patient was treated in the intensive care unit till $15^{\text {th }}$ of January 2016, but then she was transferred to P. Stradins CUH.

The patient previously had never smoked, never had oncological diseases Menses were normal. The woman had been pregnant three times and had given birth to one child. In February 2015 she had a thrombophlebitis in the left elbow pit and thrombosis was detected also in the vena saphena magna. These conditions were associated with use of oral contraceptives. Since August 2015 the patient stopped to use them. The patient received the therapy with Rivaroxaban. 
After the patient was admitted to P. Stradins CUH, other investigations were performed. Echocardiography showed at least two structures with medium echogenicity, size about $2,3 \times 0,7 \mathrm{~cm}$ and $0,9 \times 0,6 \mathrm{~cm}$ bound to chordae tendineae of the tricuspid valve leaflets. Computed tomography (CT) of the heart (Figure 1) revealed the structure in the right ventricle fixed to anterior leaflet of the tricuspid valve in the site where chordae tendineae were attached to papillary muscle. Also, CT showed bilateral pulmonary thromboembolism, interstitial inflammation in the lungs with alveolar consolidation in segments S9 and S10 of the left lung and possible pericardial damage.

Video-assisted thoracoscopic surgery was performed for biopsy of lymph nodes and marginal lung resection for histological examination that revealed primary adenocarcinoma. Histological examination performed on $4^{\text {th }}$ of February 2016 revealed poorly differentiated tubular papillary and solid metastases of the adenocarcinoma in the lymph node, invasion through the lymph node capsule and lymphatic vessels (Figure 2), visceral pleural invasion of adenocarcinoma (Figure 3), invasion in blood vessels (Figure 4) and carcinomatous lymphangitis. Tumor was composed of pleomorphic median and large size cells with light cytoplasm and marked nucleoli, with presence of psammoma bodies (Figure 5). Immunohistochemistry revealed intensively expressed cytokeratin AE1/3, cytokeratin 7 and nuclear thyroid transcription factor-1 (TTF-1) in tumor cells (Figures 6,7) and mildly expressed high molecular weight cytokeratin focally and nuclear protein p63 in small foci. Expression of cytokeratin 20, CDX20, protein S-100, HMB45, estrogen and progesterone receptors as well as neuroendocrine markers were not observed in the tumor cells. The growth fraction was detected by Ki-67 which was about $6 \%-10 \%$. A diagnosis of left lung adenocarcinoma at the stage T1N3M0, III B, grade G3 was made.

After histological diagnosis baseline CT scan was done before initiation of palliative chemotherapy. Subpleural lung lesion in left lower lobe S9, S10 was $\sim 45 \times 48 \times 39$ mm large, as well enlarged mediastinal lymph nodes were present with short axis size 12-15 mm. After 2 cycles of chemotherapy lung

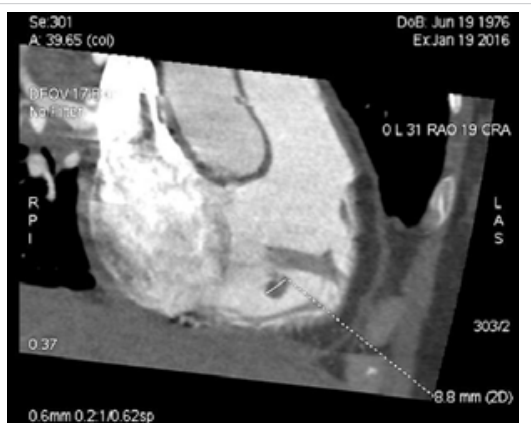

Figure 1: CT of the heart on $19^{\text {th }}$ of January 2016. Structure in the cavity of right ventricle that was fixed to anterior leaflet of the tricuspid valve in the site where chordae tendineae are attached to papillary muscle. (Image from Diagnostic Radiology Institute of P. Stradins CUH.

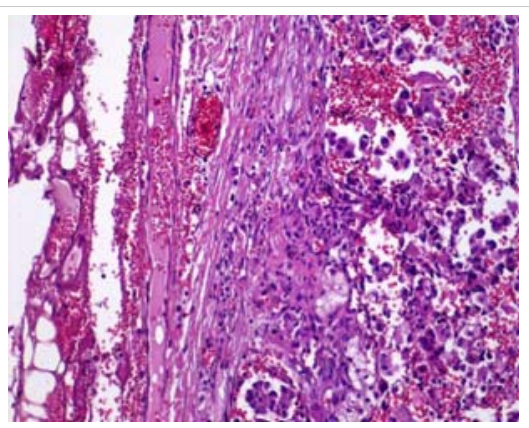

Figure 2: Poorly differentiated tubular papillary and solid metastatic adenocarcinoma in the lymph node with invasion through the lymph node capsule. Lymphangitis carcinomatosa. (x20, H\&E. Dept of Pathology, Medical faculty of the University of Latvia and Institute of Pathology of P. Stradins CUH.

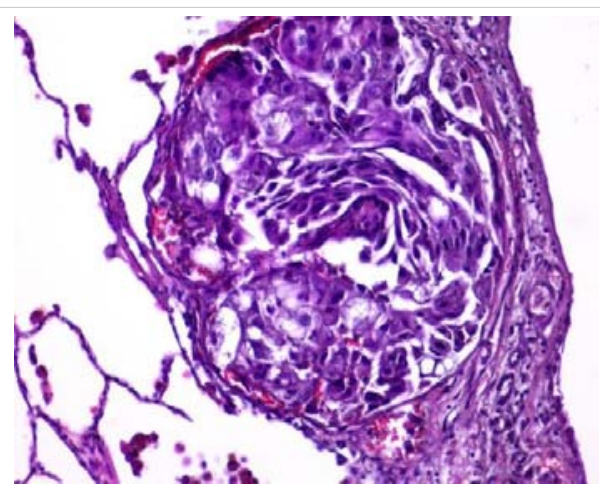

Figure 3: Visceral pleural invasion of adenocarcinoma (x20, H\&E. Dept of Pathology, Medical faculty of the University of Latvia and Institute of Pathology of P. Stradins CUH.

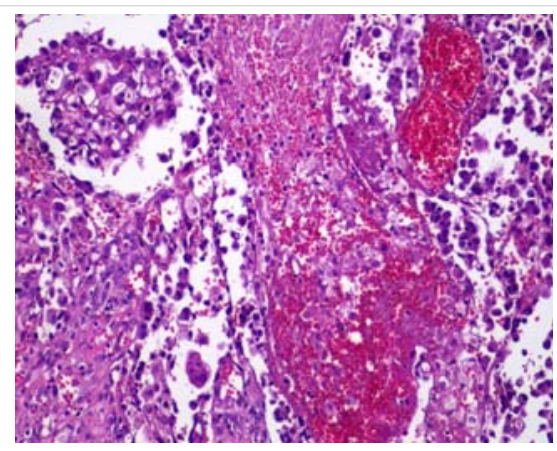

Figure 4: Dissemination of adenocarcinoma to lung tissue and blood vessel invasion (x20, H\&E. Dept of Pathology, Medical faculty of the University of Latvia and Institute of Pathology of P. Stradins CUH.

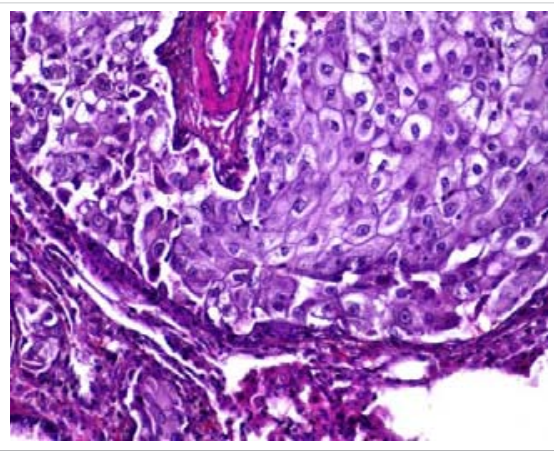

Figure 5: The tumor is composed of the pleomorphic median and large cells, predominantly with light cytoplasm and marked nucleoli, with the presence of psammoma bodies (x20, H\&E. Dept of Pathology, Medical faculty of the University of Latvia and Institute of Pathology of P. Stradins CUH. 


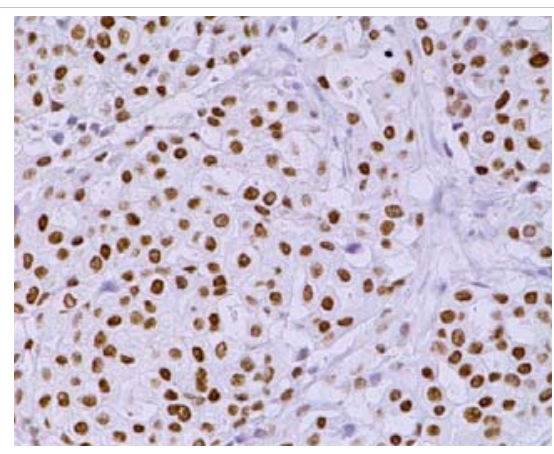

Figure 6: Immunohistochemical examination. The lymph node with metastatic adenocarcinoma of the lung. Tumor cells intensively express the organ-specific marker TTF-1 (x20, Immunohistochemistry. Dept of Pathology, Medical faculty of the University of Latvia and Institute of Pathology of P. Stradins CUH.

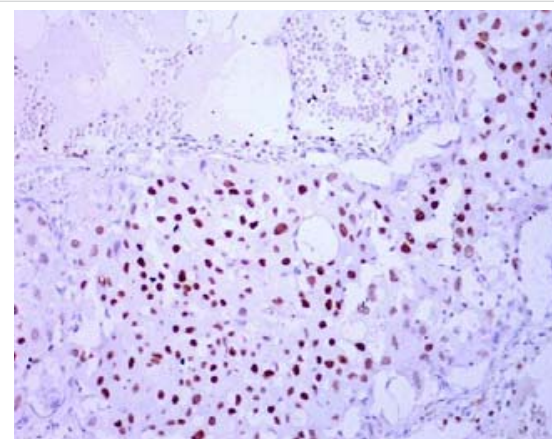

Figure 7: Immunohistochemical examination. Poorly differentiated adenocarcinoma of the lung. Tumor cells intensively express the organ-specific marker TTF-1 ( $x 20$, Immunohistochemistry. Dept of Pathology, Medical faculty of the University of Latvia and Institute of Pathology of P. Stradins CUH.

lesion decreased to long axis size $21 \mathrm{~mm}$ and mediastinal lymph nodes-to 7-10 $\mathrm{mm}$. No abnormalities were found in thyroid gland.

At the hospital due to infected thrombus in the right ventricle the patient received antibacterial therapy with Ceftriaxone $2 \mathrm{~g}$ one time a day for seven days, Gentamicin for 12 days, Piperacillin/Tazobactam 4,5 g three times a day for 17 days and Vancomycin $1 \mathrm{~g}$ two times a day for 11 days.

On $12^{\text {th }}$ February 2016, oncology council was convened which decided that antibacterial and anticoagulant therapy should be continued, control CT scan of the heart should be performed, and chemotherapy was prescribed. Tumor genetic testing was performed and none of the EGFR mutations were detected. CT of the heart was repeatedly performed, and it revealed that the thrombotic masses were resolved, and antibacterial therapy was stopped. Patient was discharged from the hospital with following recommendations:

- to continue treatment under the supervision of a general practitioner and oncologist.

- to continue therapy with low molecular weight heparin, for example Enoxaparin 0,6 ml 2 times a day subcutaneously at least for six months (in the case of problems with financing, the therapy with Warfarin is indicated); and therapy with Ivabradin 7,5 mg 2 times a day indicated.
- admission to the Oncology Clinic of P. Stradins CUH planned to $25^{\text {th }}$ of February 2016 for initiation of chemotherapy.

The woman was admitted to P. Stradins CUH on $25^{\text {th }}$ February 2016. Due to difficulty in achieving peripheral venous puncture, on $13^{\text {th }}$ of March 2016, a venous access port CELSITE was implanted. Six courses of first-line chemotherapy with Cisplatin/Pemetrexed prophylactic hemostimulating therapy during clinical trial was planned. The woman agreed to participate in the clinical study, and she was included in it starting from $3^{\text {rd }}$ of March 2016. Screening investigations including clinical analyses, lungs and abdominal CT scans were performed (Figure 8). Signs of pulmonary embolism and pathologic elements in the heart cavities were no longer observed. To reduce the toxicity of Pemetrexed therapy Folic acid $1 \mathrm{mg}$ one time a day and Cyanocobalamin $1 \mathrm{mg}$ subcutaneously every nine weeks was initiated. The patient continued treatment at the hospital till $6^{\text {th }}$ of April 2016. During hospitalization periodic febrile temperature episodes without neutropenia were observed.

On $1^{\text {st }}$ of April 2016, blood culture was obtained from the venous access port and Burkholderia cepacica microorganism sensitive to Trimethoprim-Sulfamethoxazole was revealed, so on $6^{\text {th }}$ April 2016, therapy with this antibiotic was initiated.

On $15^{\text {th }}$ of April 2016, patient was repeatedly hospitalized to P. Stradins CUH for continuation of chemotherapy. The woman felt the same as before and there were no significant changes in the blood analysis. The patient used antibacterial therapy with Trimethoprim-Sulfamethoxazole and febrile temperature was not observed. On 18 ${ }^{\text {th }}$ of April 2016 blood culture from the venous access port was repeated, and it was negative. On $27^{\text {th }}$ April 2016 antibacterial therapy with Trimethoprim-Sulfamethoxazole was stopped. Control computed tomography of the lungs and the abdomen was repeated on $18^{\text {th }}$ April 2016 (Figures 9,10). Reduction of lung tumor size was observed. However, metastasis in the vertebral body Th1 was detected, so the stage of the disease was changed to IV.

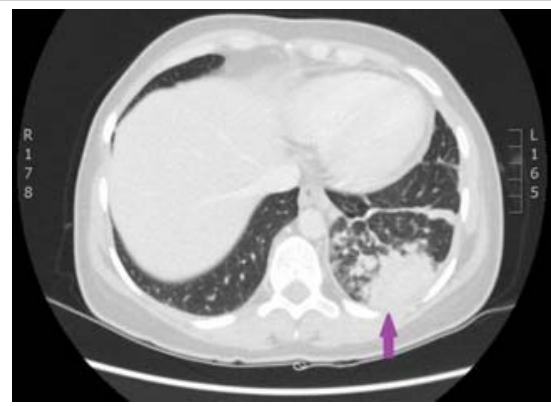

Figure 8: Computed tomography of the lungs. $4^{\text {th }}$ March 2016. Peripheral adenocarcinoma in the left lower lobe basally, Pathologic infiltrative focus in S9, and $\mathrm{S} 10$ segments. Diffuse lymphangitis in the right lung, and in lower lobe of the left lung; bilateral hilar lymphadenopathy of lungs, mediastinum and left cervical area. (Image from Diagnostic Radiology Institute of P.Stradins CUH). 
The woman was admitted to P. Stradins CUH on $6^{\text {th }}$ June 2016 for continuation of chemotherapy. Control analyses and computed tomography were performed, and positive dynamic was observed again, however septic pulmonary emboli bilaterally were observed (Figures 11,12). Growth of Burkholderia cepacica in the venous access port CELSITE was repeatedly detected, so the port was evacuated on $7^{\text {th }}$ June 2016.

On $18^{\text {th }}$ of July 2016 , control blood analyses and computed tomography were carried out after six courses of palliative chemotherapy (Figures 13,14). Since July $18^{\text {th }}, 2016$ therapy with Warfarin 7,5/10 mg once a day with Fraxiparine 0,6 ml once a day was initiated on condition that treatment with Fraxiparine would be stopped if INR would be $>2$. Also, therapy with Folic acid and injection of Cyanocobalamin were discontinued (Figures 15,16).

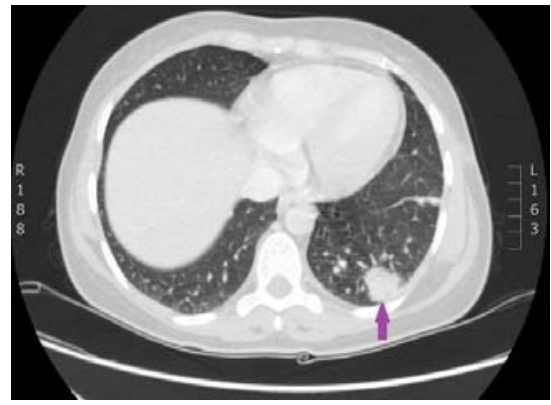

Figure 9: Computed tomography of the lungs. $18^{\text {th }}$ April 2016. Positive dynamics after therapy: local changes in the basal segment of the left lung have regressed, as well did lymphangitis, and hilar and mediastinal lymphadenopathy. Metastasis to the vertebral body, has been sclerotized after therapy (Illustration from Diagnostic Radiology Institute of P. Stradins CUH).

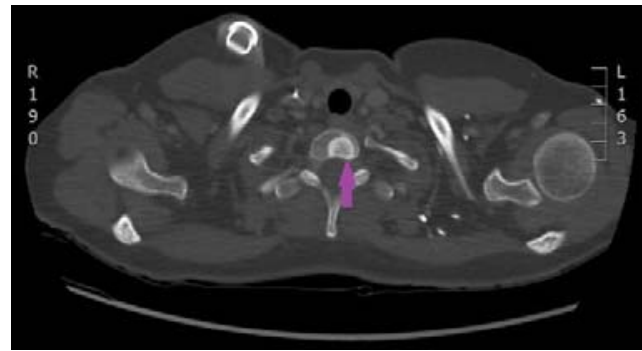

Figure 10: Computed tomography of the lungs. $18^{\text {th }}$ April 2016. Positive dynamics after therapy: local changes in the basal segment of the left lung have regressed, as well did lymphangitis, and hilar and mediastinal lymphadenopathy. Metastasis to the vertebral body, has been sclerotized after therapy (Illustration from Diagnostic Radiology Institute of P. Stradins CUH)

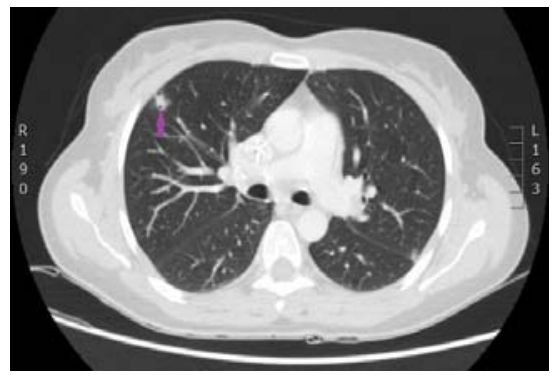

Figure 11: 11.06. 2016. Computed tomography of lungs. Presence of septic emboli in both lungs (CT scan from Diagnostic Radiology Institute of P.Stradins CUH).

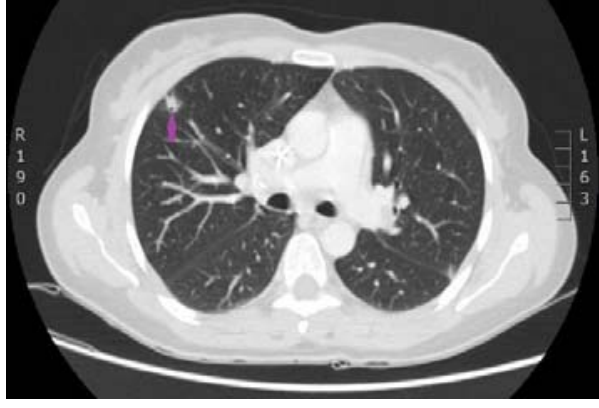

Figure 12: 11.06. 2016. Computed tomography of lungs. Presence of septic emboli in both lungs (CT scan from Diagnostic Radiology Institute of P.Stradins CUH).

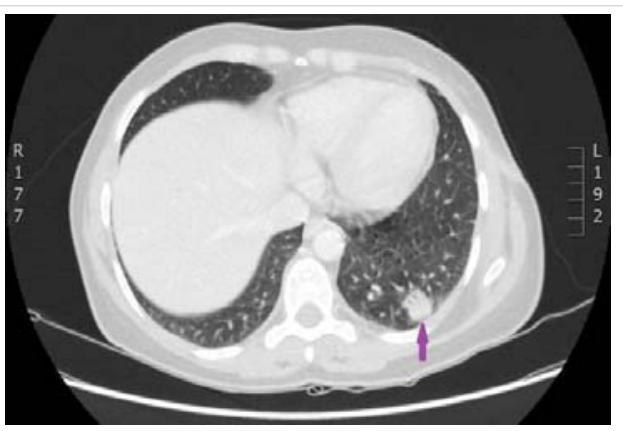

Figure 13: Control CT scan of the lungs on $18^{\text {th }}$ of July 2016. Signs of partial remission after 6 cycles of therapy with Cisplatin/Pemetrexed. Septic emboli in both lungs have disappeared (CT scan from Diagnostic Radiology Institute of P.Stradins CUH).

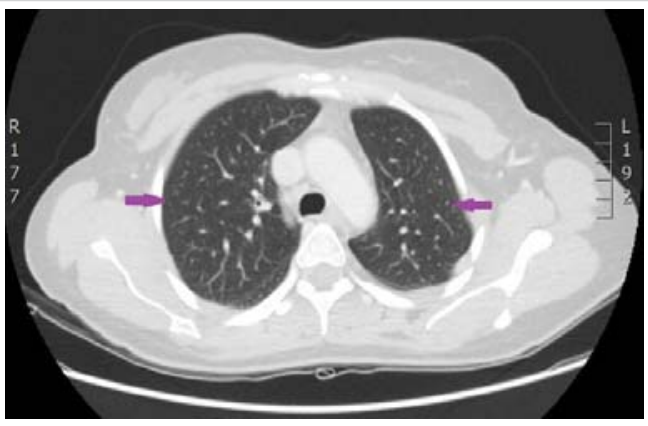

Figure 14: Control CT scan of the lungs on $18^{\text {th }}$ of July 2016. Signs of partial remission after 6 cycles of therapy with Cisplatin/Pemetrexed. Septic emboli in both lungs have disappeared (CT scan from Diagnostic Radiology Institute of P.Stradins $\mathrm{CUH}$ ).

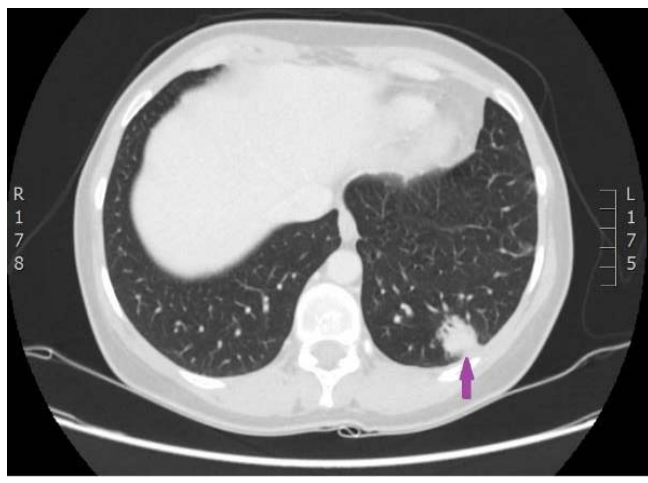

Figure 15: 29.08.2016. Computed tomography of the lungs. Compared with the previous examination, performed on 18.07.2016, the size of the structure in the left lower lobe has increased in all planes (maximal diameter in the axial plane is $23 \mathrm{~mm}$ ), and new subpleural intrapulmonary lymph nodes (9 and $12 \mathrm{~mm}$ ) near the mediastinal pleura have appeared. (CT scan from Diagnostic Radiology Institute of P.Stradins CUH). 


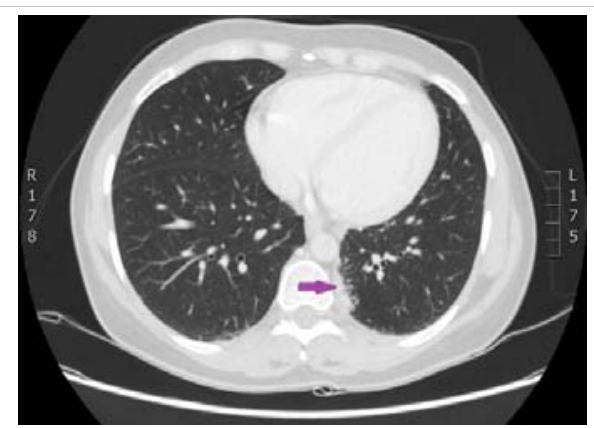

Figure 16: C29.08.2016. Computed tomography of the lungs. Compared with the previous examination, performed on 18.07.2016, the size of the structure in the left lower lobe has increased in all planes (maximal diameter in the axial plane is $23 \mathrm{~mm}$ ), and new subpleural intrapulmonary lymph nodes (9 and $12 \mathrm{~mm}$ ) near the mediastinal pleura have appeared. (CT scan from Diagnostic Radiology Institute of P.Stradins CUH)

The woman stopped injection of Fraxiparine on $25^{\text {th }}$ July 2016 and continued to use Warfarine $8,5 \mathrm{mg}$ one time a day.

One and a half months after cessation of Cisplatin/ Pemetrexed chemotherapy courses progression of the adenocarcinoma was detected, therefore the second line of chemotherapy with Docetaxel which included two courses was initiated .

On $9^{\text {th }}$ November 2016, the patient again was admitted to P.Stradins CUH for performing control computed tomography after receiving two courses of the second line of chemotherapy (Figures 17-19). The woman's condition got worse-chest discomfort and dyspnea on exertion appeared. On $11^{\text {th }}$ November 2016, superficial vein thrombosis of the lower limbs was detected despite the therapy with Warfarin, therefore injections of Fraxiparine were renewed. The third line chemotherapy with two courses of Carboplatin/Etoposide was planned.

On $29^{\text {th }}$ of December 2016 the woman was hospitalized to P.Stradins CUH for performing control CT of lungs and abdomen to decide whether to continue the third line of chemotherapy. Physical examinations revealed slightly harder supraclavicular lymph nodes bilaterally, however their size was normal. On $29^{\text {th }}$ December 2016, computed tomography of the lungs and the abdomen was performed which showed progressive lymphangitis, new subpleural metastases (Figures 20-22) why the third line of chemotherapy was discontinued. The tumor sample was tested for ROS1 mutation using NGS methodology. Examination found SLC34A2-ROS1 fusion and excluded mutations of EGF, KRAS, ALK, BRAF, MET, RET, ERBB2 genes.

Since $1^{\text {st }}$ January 2017 the woman had complaints of increased cough, which was observed more often through the day, when moving. When coughing, the patient had chest pain on the left side. Physical examination revealed supraclavicular specific lymph nodes on both sides (on the left side > than on the right side).

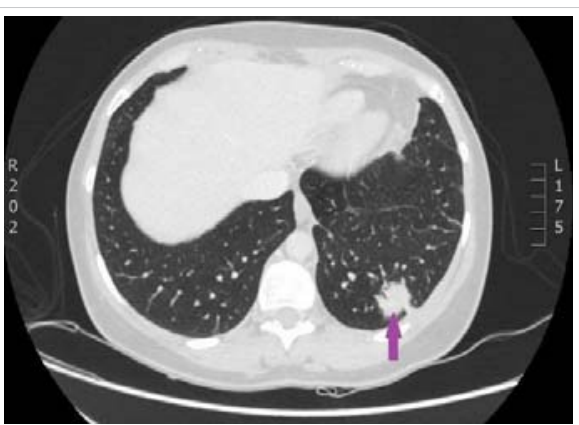

Figure 17: CT scan of the lungs on $9^{\text {th }}$ of November 2016. Compared with the previous examination, negative changes of the process are seen. Pathological node in the lower lobe of the left lung compared to the previous examination, has increased, its maximal diameter in the axial plane is $24 \mathrm{~mm}$; in $\mathrm{S} 10$ paramediastinaly infiltrative focal structure with maximal diameter of $26 \mathrm{~mm}$ is present that infiltrates the costal pleura, there is pathological lymph node beside the infiltrative focal structure in the mediastinum, its transverse size in the axial plane is $12 \mathrm{~mm}$. Lymphangitis has progressed in the upper lobe of the right lung; enlarged lymph nodes are present in the mediastinum. Small nodules are present on the right intralobar, costal and diaphragmatic pleura and similar nodules on the diaphragmatic pleura in the left side. (CT scans from Diagnostic Radiology Institute of P.Stradins CUH).

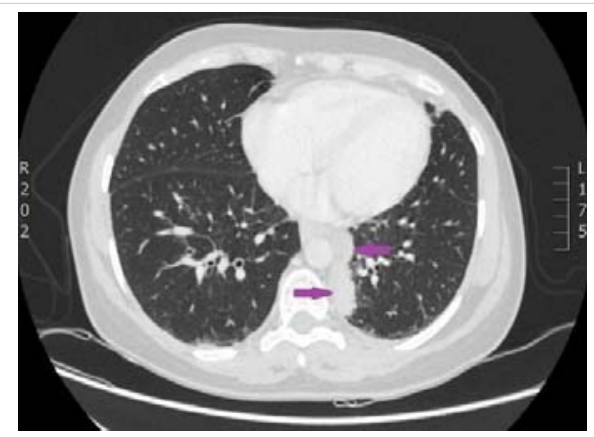

Figure 18: CT scan of the lungs on $9^{\text {th }}$ of November 2016. Compared with the previous examination, negative changes of the process are seen. Pathological node in the lower lobe of the left lung compared to the previous examination, has increased, its maximal diameter in the axial plane is $24 \mathrm{~mm}$; in $\mathrm{S} 10$ paramediastinaly infiltrative focal structure with maximal diameter of $26 \mathrm{~mm}$ is present that infiltrates the costal pleura, there is pathological lymph node beside the infiltrative focal structure in the mediastinum, its transverse size in the axial plane is $12 \mathrm{~mm}$. Lymphangitis has progressed in the upper lobe of the right lung; enlarged lymph nodes are present in the mediastinum. Small nodules are present on the right intralobar, costal and diaphragmatic pleura and similar nodules on the diaphragmatic pleura in the left side. (CT scans from Diagnostic Radiology Institute of P.Stradins CUH).

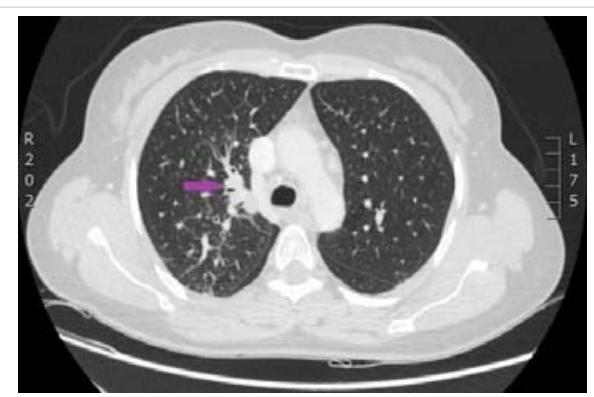

Figure 19: CT scan of the lungs on $9^{\text {th }}$ of November 2016. Compared with the previous examination, negative changes of the process are seen. Pathological node in the lower lobe of the left lung compared to the previous examination, has increased, its maximal diameter in the axial plane is $24 \mathrm{~mm}$; in $\mathrm{S} 10$ paramediastinaly infiltrative focal structure with maximal diameter of $26 \mathrm{~mm}$ is present that infiltrates the costal pleura, there is pathological lymph node beside the infiltrative focal structure in the mediastinum, its transverse size in the axial plane is $12 \mathrm{~mm}$. Lymphangitis has progressed in the upper lobe of the right lung; enlarged lymph nodes are present in the mediastinum. Small nodules are present on the right intralobar, costal and diaphragmatic pleura and similar nodules on the diaphragmatic pleura in the left side. (CT scans from Diagnostic Radiology Institute of P.Stradins CUH). 


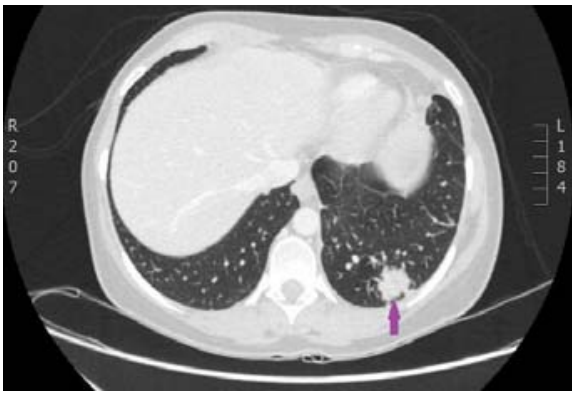

Figure 20: CT scan of the lungs on $29^{\text {th }}$ of December 2016. Lymphangitis has progressed in the upper lobe of the right lung; new subpleural metastases are seen. (Images from Diagnostic Radiology Institute of P. Stradins CUH).

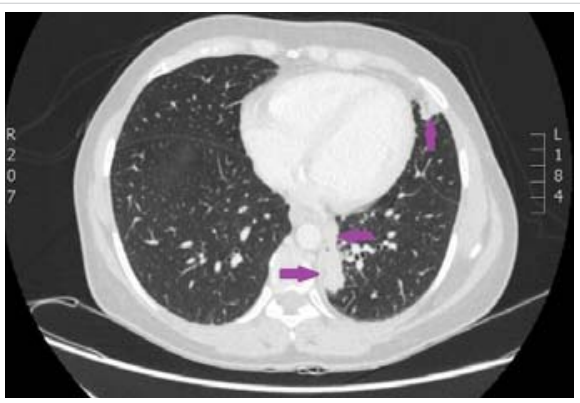

Figure 21: CT scan of the lungs on $29^{\text {th }}$ of December 2016. Lymphangitis has progressed in the upper lobe of the right lung; new subpleural metastases are seen. (Images from Diagnostic Radiology Institute of P. Stradins CUH).

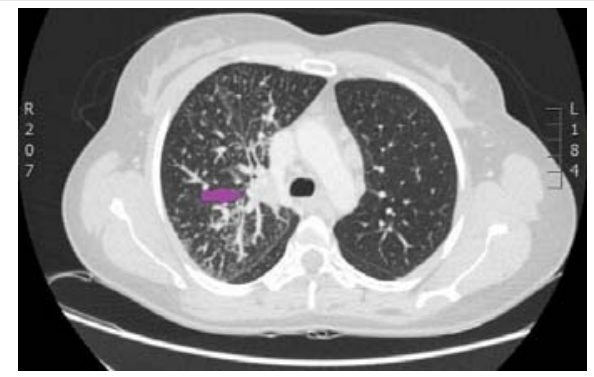

Figure 22: CT scan of the lungs on $29^{\text {th }}$ of December 2016. Lymphangitis has progressed in the upper lobe of the right lung; new subpleural metastases are seen. (Images from Diagnostic Radiology Institute of P. Stradins CUH).

Due to the detected ROS mutation, a decision was made about therapy with Crizotinib which the patient used one month from $6^{\text {th }}$ April 2017 to $6^{\text {th }}$ May 2017. Rapid improvement was observed clinically after the first week of treatment.

On $10^{\text {th }}$ of May 2017 the specific supraclavicular lymph nodes disappeared. Unfortunately, because of the problems in financing, the patient did not continue the medication.

On 29th May 2017, due to unconsciousness, computed tomography of the head was performed in patient's regional hospital and multiple brain metastases were detected (Figure 23). On $30^{\text {th }}$ of May 2017, the patient was transferred to P. Stradins CUH. It was stated that the patient was unconscious, she was not able to communicate with personal and and respond to commands. Patient's condition was serious. Rehydration therapy and other types of symptomatic therapy were initiated but without an effect. The patient's condition dynamically worsened, carcinomatous intoxication

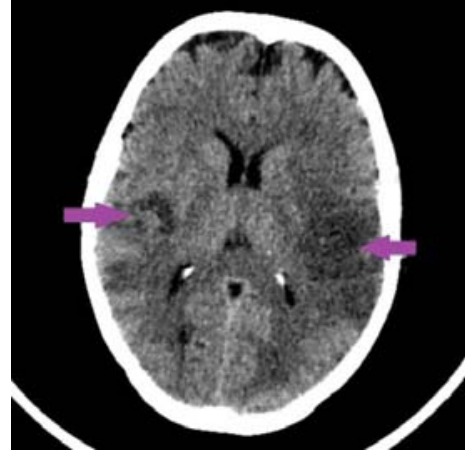

Figure 23: 29.05.2017. CT of the head. Pathological changes like circumscribed changes in radiodensity with predominantly decreased density are present in both parietal lobes, more manifested in the left hemisphere; additionally, hardly recognizable small focuses of increased radiodensity are present. (IImages from the Department of Radiology of association of Balvi and Gulbene hospital).

progressed, and signs of cerebral edema appeared. On $10^{\text {th }}$ of June 2017 at 01.00 p.m. death occurred.

\section{Discussion}

The described case of the disease of the 39-year-old nonsmoking woman, which ended with a fatal outcome one and a half years after the diagnosis of pulmonary adenocarcinoma was established, revealed the question whether it was possible to save her life or to extend her survival.

A year before the onset of the first symptoms that could be associated with lung tumor, thrombophlebitis of the upper extremities and phlebothrombosis of lower extremities had appeared. Thrombosis was associated with the use of contraceptives. Today we can interpret these changes as paraneoplastic syndrome, which manifested itself in the form of thrombophilia. It is well known that paraneoplastic syndromes can appear as early as a year before the tumor is detected. In this case the patient had no signs of bronchocarcinoma at the beginning of her sickness.

The reason why the patient was looking for medical care was also associated with thrombosis and pulmonary thromboembolism. Correct diagnosis-pulmonary adenocarcinoma was established relatively quickly after videoassisted thoracoscopic surgery, pulmonary resection and lymph node extirpation with subsequent histological investigation.

The material of tumor tissue was examined in more detail using immunohistochemistry and genetic methods. A ROS1 mutation was revealed only after two ineffective chemotherapy courses, 11 months after histological identification of the adenocarcinoma. Specific therapy with Crizotinib, a ROS inhibitor, was initiated four months after detection of ROS1 mutation, when the disease had already entered the stage IV. Despite the late stage of the disease, one week after treatment with Crizotinib, the specific supraclavicular lymph nodes disappeared, and clinical improvement was observed. Unfortunately, therapy was discontinued after a month due to financial problems but two months later the patient died. 
Would the specific therapy have been effective if treatment had been started at an earlier stage of the disease?

A clinical case in Salzburg hospital has been described when a complete remission was achieved after treatment with Crizotinib. A non-smoking 30-year-old woman with pulmonary adenocarcinoma in Stage IV received four cycles of chemotherapy with Pemetrexed but positive dynamic was not observed. Additional examination of tumor tissue revealed ROS1 mutation. Treatment with Crizotinib was started six months after the diagnosis of the tumor was made. Eight weeks after the initiation of specific therapy, complete remission was achieved - positron emission tomographycomputed tomography was performed, and it did not reveal tumor-specific tissue. The patient continued to use Crizotinib $250 \mathrm{mg}$ once a day and progression of the disease was not observed for the following four months [25].

The described case shows a high effectiveness of specific therapy with Crizotinib despite late treatment initiation. Also in our clinical case, positive dynamic was observed at the beginning when the specific treatment was initiated. Unfortunately, therapy was started at the late stage of the disease.

The analysis of these cases shows that, when adenocarcinoma is detected in early-aged non-smoking individuals, genetic tests of tumor tissue should be carried out as soon as possible to identify ROS1 as well as EGFR, ALK, MET, HER2, BRAF, RET and other gene mutations with next-generation sequencing techniques. In case of a positive result, specific therapy with a ROS1 tyrosine kinase receptors inhibitor, such as Crizotinib, should be initiated immediately.

Also, treating the patients who have primary thrombophilia, consideration should be given to the possible presence of paraneoplastic syndrome and the careful assessment of patient's symptoms that might indicate on possible tumor.

\section{Conclusion}

In cases when pulmonary adenocarcinoma is detected in young individuals, especially in non-smoking women, it is necessary to exclude ROS1 mutation as soon as possible.

If ROS1 mutation is found, treatment with ROS1 receptor tyrosine kinase inhibitors such as Crizotinib should be initiated without delay.

In the case of primary thrombophilia, the possibility of paraneoplastic syndrome should be taken into account.

\section{References}

1. Bray F, Ferlay J, Soerjomataram I, Siegel RL, Torre LA, et al. Global cancer statistics 2020: GLOBOCAN estimates of incidence and mortality worldwide for 36 cancers in 185 countries. CA Cancer J Clin. 2021; 0: 1-41.

2. Islami F, Torre L.A, Jemal A. Global trends of lung cancer mortality and smoking prevalence. Transl Lung Cancer Res. 2015; 4: 327-338. PubMed: https://pubmed.ncbi.nlm.nih.gov/26380174/

3. Ridge CA, McErlean AM, Ginsberg MS. Epidemiology of lung cancer. Semin Intervent Radiol. 2013; 30: 93-98.

PubMed: https://pubmed.ncbi.nlm.nih.gov/24436524/

4. Barrera-Rodriguez R, Morales-Fuentes $\mathrm{J}$. Lung cancer in women. Lung Cancer (Auckl). 2012; 3: 79-89.

PubMed: https://pubmed.ncbi.nlm.nih.gov/28210127/

5. SPCK. Statistiskas dati par 2017. gadu. Onkoloǵija. 2017. https://www. spkc.gov.lv/lv/statistikas-dati

6. Davies KD, Doebele RC. Molecular Pathways: ROS1 Fusion Proteins in Cancer. Clin Cancer Res. 2013; 19: 4040-4045.

PubMed: https://pubmed.ncbi.nlm.nih.gov/23719267/

7. Bergethon K, Shaw AT, Ou SH I, Katayama R, Lovly CM, et al. ROS1 ROS1 rearrangements define a unique molecular class of lung cancers. 2012; 30: 863-870.

PubMed: https://pubmed.ncbi.nlm.nih.gov/22215748/

8. Marchetti A, Barberis M, Di Lorito A, Pace MV, Chiara MV, et al. ROS1 Gene Fusion in Advanced Lung Cancer in Women: A Systematic Analysis, Review of the Literature, and Diagnostic Algorithm. JCO Precision Oncol. 2017; 1.

9. Bubendorf L, Büttner R, Al-Dayel F, Dietel M, Elmberger G, et al. Testing for ROS1 in non-small cell lung cancer: a review with recommendations. Virchows Arch. 2016; 469: 489-503.

PubMed: https://pubmed.ncbi.nlm.nih.gov/27535289/

10. Shu Y, Li H, Shang H, Chen J, Su X, et al. Identification of a Novel MPRIP-ROS1 Fusion and Clinical Efficacy of Crizotinib in an Advanced Lung Adenocarcinoma Patient: A Case Report. Onco Targets Ther. 2020; 13: 10387-10391.

PubMed: https://pubmed.ncbi.nlm.nih.gov/33116618/

11. Solomon B. Validating ROS1 rearrangements as a therapeutic target in non-small-cell lung cancer. J Clin Onco. 2015; 33: 972-974.

PubMed: https://pubmed.ncbi.nIm.nih.gov/25667277/

12. Jun HJ, Johnson H, Bronson RT, de Feraudy S, White F, et al. The oncogenic lung cancer fusion kinase CD74-ROS activates a novel invasiveness pathway through E-Syt1 phosphorylation. Cancer Res. 2012; 72: 3764-3774.

PubMed: https://pubmed.ncbi.nlm.nih.gov/22659450/

13. Sholl LM, Sun H, Butaney M, Zhang C, Lee C, et al. ROS1 immunohistochemistry for detection of ROS1-rearranged lung adenocarcinomas. Am J Surg Patho. 2013; 37: 1441-1449. PubMed: https://pubmed.ncbi.nlm.nih.gov/23887156/

14. Shaw AT, Ou SHI, Bang YJ, Camidge DR, Dolomon SJ, et al. Crizotinib in ROS1-rearranged non-small-cell lung cancer. N Engl J Med. 2014; 371: 1963-1971.

PubMed: https://pubmed.ncbi.nlm.nih.gov/25264305/

15. Kumar A, Shanthi V, Ramanathan K. Structural and Functional Impact of G2032R Mutation in ROS1-A Theoretical Perspective. Asian J Pharmaceut Clin Res. 2017; 10: 339-344.

16. Sculier JP, Fry W. Pathology of Lung Cancer. Malignant Tumors of the Lung. 2004; 8th ed. 39-55.

17. Quaia E. Radiologic-pathologic correlations between intratumoral macroscopic and microscopic components in lung neoplasms. Congress ECR. 2006; c-186.

18. Qian X, Fu M, Zheng J, Zhou J, Zhou J. Driver Genes Associated with the Incidence of Venous Thromboembolism in Patients With NonSmall-Cell Lung Cancer: A Systematic Review and Meta-Analysis. Front Oncol. 2021; 11: 680191.

PubMed: https://pubmed.ncbi.nlm.nih.gov/33996610/

19. Chiari R, Ricciuti B, Landi L, Morelli AM, Delmonte A, et al. ROS1rearranged Non-small-cell Lung Cancer is Associated With a High Rate 
of Venous Thromboembolism: Analysis From a Phase II, Prospective, Multicenter, Two-arms Trial (METROS). 2020; 21:15-20.

PubMed: https://pubmed.ncbi.nIm.nih.gov/31607443/

20. Liu Y, Wang W, Wu F, Gao G, Xu J, et al. High discrepancy in thrombotic events in non-small cell lung cancer patients with different genomic alterations. Transl Lung Cancer Res. 2021; 10: 1512-1524.

PubMed: https://pubmed.ncbi.nlm.nih.gov/33889526/

21. Kanaji N, Watanabe N, Kita N, Bandoh S, Tadokoro A, et al Paraneoplastic syndromes associated with lung cancer. World J Clin Oncol. 2014; 5: 197-223.

PubMed: https://pubmed.ncbi.nIm.nih.gov/25114839/

22. Marinho FC. de A, Takagaki TY. Hypercoagulability and lung cancer.
J Bras Pneumol. 2008; 34: 312-322.

PubMed: https://pubmed.ncbi.nlm.nih.gov/18545828/

23. Falanga A. The Cancer-Thrombosis Connection. The Hematologist: ASH News \& Reports. 2011; 8: 4-5.

24. Boccaccio C, Comoglio PM. Genetic link between cancer and thrombosis. J Clin Oncol. 2009; 27: 4827-4833.

PubMed: https://pubmed.ncbi.nlm.nih.gov/19738115/

25. Blaukovitsch M, Hofer J, Hutarew G, Müllauer L, Ingrid SK, et al. ROS1 mutation and treatment with Crizotinib in a 30-yearv old Caucasian woman with stage IV non-small cell lung cancer/adenocarcinoma and complete remission. Thorac Cancer. 2014; 5: 455-459. PubMed: https://pubmed.ncbi.nlm.nih.gov/26767038/ 